\title{
Expression and clinicopathological implication of DcR3 in lung cancer tissues: a tissue microarray study with 365 cases
}

\author{
Yu Zhangl,* \\ Jie Luo ${ }^{2, *}$ \\ Rongquan $\mathrm{He}^{3}$ \\ Wenting Huang' \\ Zuyun $\mathrm{Li}^{\prime}$ \\ Ping $\mathrm{Li}^{\prime}$ \\ Yiwu Dang' \\ Gang Chen' \\ Shikang $\mathrm{Li}^{4}$ \\ 'Department of Pathology, \\ 2Department of Medical Oncology, \\ First Affiliated Hospital of Guangxi \\ Medical University, ${ }^{3}$ Center for \\ Genomic and Personalized Medicine, \\ Guangxi Medical University, \\ ${ }^{4}$ Department of Thoracic and \\ Cardiovascular Surgery, First \\ Affiliated Hospital of Guangxi Medical \\ University, Nanning, Guangxi, People's \\ Republic of China \\ *These authors contributed equally \\ to this work
}

This article was published in the following Dove Press journal:

OncoTargets and Therapy

10 August 2016

Number of times this article has been viewed

Background: Decoy receptor 3 (DcR3) has been reported to be involved in different cancers. However, few related researches have been accomplished on the role of DcR3 in lung cancer. Objective: To explore the expression level and clinicopathological implication of DcR3 protein in lung cancer tissues.

Materials and methods: Immunohistochemistry was used to examine DcR3 protein expression in lung cancer $(n=365)$ and normal lung tissues $(n=26)$. The relationships between DcR3 expression and clinical parameters were further investigated. Furthermore, the diagnostic and clinicopathological value of DcR3 mRNA was analyzed based on The Cancer Genome Atlas database in lung cancer patients.

Results: Compared to normal lung tissues, DcR3 expression was significantly higher in lung cancer ( $P=0.007)$ tissues, including small-cell lung cancer $(P=0.001)$ and non-small-cell lung cancer $(P=0.008)$. In addition, DcR3 expression was related to tumor-node-metastasis (TNM) stage $(P<0.001)$, tumor diameter $(P=0.007)$, distant metastasis $(P<0.001)$, and lymph node metastasis $(P<0.001)$ in lung cancers. When concerning non-small-cell lung cancer, consistent correlations between DcR3 expression and TNM stage $(P<0.001)$, tumor diameter $(P=0.019)$, distant metastasis $(P<0.001)$, and lymph node metastasis $(P<0.001)$ were found. Simultaneously, in small-cell lung cancer, TNM stage $(P=0.004)$ and lymph node metastasis $(P=0.005)$ were also associated with DcR3 expression. Additionally, receiver operator characteristic curve revealed that the area under curve (AUC) of DcR3 was 0.637 (95\% confidence interval [CI] 0.531-0.742) for lung cancer. Furthermore, DcR3 was overexpressed in both adenocarcinoma and squamous cell carcinoma tissues than in noncancerous lung tissues (all $P<0.0001$ ) based on the data from The Cancer Genome Atlas. AUC of DcR3 was 0.726 (95\% CI 0.644-0.788) for lung adenocarcinoma patients and 0.647 (95\% CI 0.566-0.728) for squamous cell carcinoma patients. DcR3 expression was also related to the overall survival $(P<0.001)$ and disease-free survival $(P<0.001)$ of lung adenocarcinoma according to the data from The Cancer Genome Atlas.

Conclusion: Our study confirms that DcR3 might be involved in the tumorigenesis and deterioration of lung cancer. Therefore, the detection of DcR3 gains the potential to be applied in the clinic for screening and progression prediction of lung cancer.

Keywords: DcR3, lung cancer, immunohistochemistry, prognosis, TCGA

\section{Introduction}

Lung cancer is the most common cancer and the first leading cause of cancer-related death worldwide. ${ }^{1,2}$ Over 1.8 million lung cancer patients were diagnosed per year, accounting for $\sim 13 \%$ of total new cancer cases. ${ }^{3}$ Lung cancer is divided into two categories based on the histological type: small-cell lung cancer (SCLC) and non-small-cell lung 
cancer (NSCLC). Among these two types, NSCLC accounts for $80 \%-85 \%$ of the newly diagnosed lung cancer cases. More than $70 \%$ of the NSCLC cases are in the advanced stage of the disease, and the 5-year survival rate for NSCLC is only $16 \%{ }^{4}$ In the management of locally advanced or stage I lung cancer, surgery is the best option. In stage II/IIIA lung cancer, surgery or the combination with chemoradiotherapy might be the standard adjuvant approach. For late-stage (IIIB/IV) lung cancer, platinum-based combined chemoradiotherapy is the current standard treatment, which still has a great developing space. ${ }^{5,6}$ Hence, the research for efficient molecular biomarkers is of great significance to the diagnosis and treatment of lung cancer.

Decoy receptor 3 (DcR3), also identified as TNF family receptor 6, tumor necrosis factor receptor super family member 6B, or M68, is located on chromosome 20q13.3. ${ }^{7-10}$ Some studies have provided evidence for the overexpression of DcR3 in malignant tumors, such as renal cell carcinoma, hepatocellular carcinoma, glioma, and colorectal carcinoma. ${ }^{11-14}$ Additionally, DcR3 was confirmed to be associated with some biological functions, for example, proliferation, invasion, metastasis, and apoptosis, ${ }^{11,12}$ which might be related to immune evasion by malignant tumors. ${ }^{15}$

Till date, only three studies related to the role of DcR3 in lung cancer have been published. Pitti et $\mathrm{al}^{14}$ first reported the discovery of a soluble decoy receptor and named it as DcR3. They found that $D c R 3$ gene was highly expressed in six out of 15 lung tumors. Sung et $\mathrm{al}^{16}$ explored the role of $D c R 3$ gene expression by comparing two different lung cancer cell lines. They found that DcR3 could inhibit p53-dependent apoptosis by regulating cellular response to ionizing radiation. Wu et $\mathrm{al}^{17}$ used enzyme-linked immunosorbent assay to measure DcR3 serum levels in several malignant cancers, including lung cancer. They disclosed that a $10 \%$ positive incidence of DcR3 was detected in lung adenocarcinomas.
In our previous work, we had studied the role of DcR3 in glioma, gastric cancer, and hepatocellular carcinoma. ${ }^{12,18-21}$ However, the expression and clinicopathological implication of DcR3 in lung cancer tissues was still unclarified; hence, we designed this study to further explore the correlation between DcR3 protein level and lung cancer.

\section{Materials and methods}

\section{Study design on clinical samples}

A total of 365 tumor and 26 normal lung tissues were included in this retrospective study. All these lung tissues were constructed into three microarrays. All lung cancer tissues were collected from the First Affiliated Hospital of Guangxi Medical University, People's Republic of China (from January 2010 to December 2012). All these cases were ensured to be selected randomly from initial pneumonectomies without treatment. The study protocol was approved by the Ethical Committee of the First Affiliated Hospital of Guangxi Medical University. Written informed consent was obtained from the patients and clinicians for the usage of the samples for research. The lung cancer and normal lung patients were aged 19-84 and 19-73 years, with a mean age of 57.67 and 54.03 years, respectively. Of these, 26 cases were SCLC and 339 were NSCLC. Among the NSCLC cases, 127 were adenocarcinomas, 175 were squamous cell carcinomas, 28 were adenosquamous carcinomas, eight were undifferentiated carcinomas, and one was large cell carcinoma (Table 1). All these tissues were annotated for DcR3 expression by two pathological doctors independently without knowing patient information. All the clinicopathological information was provided by medical records and summarized in Tables $1-4$.

\section{Evaluation of immunostaining}

Expression of DcR3 was detected by immunohistochemistry (IHC). The DcR3 antibody (C-15, 1:100 dilution) was supplied

Table I Expression of DcR3 protein in lung cancer and normal lung tissues

\begin{tabular}{|c|c|c|c|c|c|}
\hline Tissue & Case & DcR3-positive, n (\%) & DcR3-negative, n (\%) & $\mathbf{Z}$ & $P$-value \\
\hline Normal lung tissue & 26 & $7(15.4)$ & $19(84.6)$ & & \\
\hline Lung cancer & 365 & $196(53.7)$ & $169(46.3)$ & 2.895 & 0.007 \\
\hline \multicolumn{6}{|l|}{ Histology } \\
\hline SCLC & 26 & $19(73.1)$ & $7(26.9)$ & 3.679 & 0.001 \\
\hline NSCLC & 339 & $180(53.1)$ & 159 (46.9) & 2.821 & 0.008 \\
\hline Adenocarcinoma & 127 & $72(56.7)$ & $55(43.3)$ & 2.821 & 0.005 \\
\hline Squamous cell carcinoma & 175 & $85(48.6)$ & $90(51.4)$ & 2.244 & 0.031 \\
\hline Adenosquamous carcinoma & 28 & $17(60.7)$ & II (39.3) & 2.605 & 0.012 \\
\hline Undifferentiated carcinoma & 8 & $6(75)$ & $2(25)$ & 2.615 & 0.013 \\
\hline Large cell carcinoma & I & 0 & I (100) & & \\
\hline
\end{tabular}

Abbreviations: DcR3, decoy receptor 3; SCLC, small-cell lung cancer; NSCLC, non-small-cell lung cancer. 
Table 2 Differential expression of DcR3 protein in relation to other clinicopathological parameters in lung cancer

\begin{tabular}{|c|c|c|c|c|c|}
\hline Tissue & Case & DcR3-positive, n (\%) & DcR3-negative, n (\%) & $\mathbf{Z}$ & $P$-value \\
\hline \multicolumn{4}{|l|}{ Age (years) } & -0.368 & 0.713 \\
\hline$<60$ & 196 & $107(54.6)$ & $89(45.4)$ & & \\
\hline$\geqq 60$ & 169 & 89 (52.7) & $80(47.3)$ & & \\
\hline \multicolumn{4}{|l|}{ Sex } & 1.264 & 0.208 \\
\hline Male & 275 & $|4|(5 \mid .3)$ & $134(48.7)$ & & \\
\hline Female & 90 & $53(58.9)$ & $37(4 I . I)$ & & \\
\hline \multicolumn{4}{|l|}{ TNM } & 4.893 & $<0.001$ \\
\hline | or II & 299 & I 44 (48.2) & $155(5 \mid .8)$ & & \\
\hline III or IV & 66 & $5 \mathrm{I}(77.3)$ & $15(22.7)$ & & \\
\hline \multicolumn{4}{|c|}{ Tumor diameter $(\mathrm{cm})$} & 2.790 & 0.007 \\
\hline$\leq 7$ & 314 & $160(51.0)$ & $154(49.0)$ & & \\
\hline$>7$ & 51 & $36(70.6)$ & $15(29.4)$ & & \\
\hline \multicolumn{4}{|c|}{ Distant metastasis } & -6.202 & $<0.001$ \\
\hline Yes & 16 & $15(93.8)$ & $\mathrm{I}(6.2)$ & & \\
\hline No & 349 & $180(5 \mid .6)$ & $169(48.4)$ & & \\
\hline \multicolumn{4}{|c|}{ Lymph node metastasis } & -8.178 & $<0.001$ \\
\hline Yes & 128 & $102(79.7)$ & $26(20.3)$ & & \\
\hline No & 237 & $96(40.5)$ & $|4|(59.5)$ & & \\
\hline
\end{tabular}

Abbreviations: DcR3, decoy receptor 3; TNM, tumor-node-metastasis.

by Santa Cruz Biotechnology Inc. (Dallas, TX, USA). Immunohistochemical staining reagents were purchased from Shanghai Changdao Biotech Co., Ltd (Shanghai, People's Republic of China). The IHC assay was performed according to the manufacturer's instructions. Two pathologists (Ping $\mathrm{Li}$ and Zuyun $\mathrm{Li}$ ) scored the average percentage of positive cells as zero $(0 \%)$, one $(1 \%-25 \%)$, two $(26 \%-50 \%)$, three $(51 \%-75 \%)$, and four $(76 \%-100 \%)$. The intensity of staining was recorded as zero (negative), one (weak), two (moderate), and three (strong). Finally, the pathological results of each sample were calculated by multiplying the scores obtained for the percentage of staining area and intensity of staining. The results were confirmed as positive for staining when the scores were more than two.

\section{Extra information regarding the effect of DcR3 on lung cancer from TCGA}

The Cancer Genome Atlas (TCGA) is a collection of exome sequencing, DNA methylation, single nucleotide polymorphism (SNP) array, miRNA-Seq, and RNA-Seq data. ${ }^{22}$ Also, TCGA could be used to analyze complicated clinical profiles and cancer genomics. ${ }^{23,24}$ The cBio Cancer Genomics Portal

Table 3 The status of DcR3 with respect to other clinicopathological parameters in NSCLC

\begin{tabular}{|c|c|c|c|c|c|}
\hline NSCLC & Case & DcR3-positive, n (\%) & DcR3-negative, n (\%) & $Z$ & $P$-value \\
\hline \multicolumn{4}{|l|}{ Age (years) } & 0.659 & 0.511 \\
\hline$<60$ & 181 & $84(46.4)$ & $97(53.6)$ & & \\
\hline$\geqq 60$ & 158 & $79(50)$ & $79(50)$ & & \\
\hline \multicolumn{4}{|l|}{ Sex } & 1.693 & 0.093 \\
\hline Male & 255 & $128(50.2)$ & I 27 (49.8) & & \\
\hline Female & 84 & $51(60.7)$ & $33(39.3)$ & & \\
\hline \multicolumn{4}{|l|}{ TNM } & 4.468 & $<0.001$ \\
\hline I or II & 286 & $138(48.3)$ & | $48(5 \mid .7)$ & & \\
\hline III or IV & 53 & $4 \mathrm{I}(77.4)$ & $12(22.6)$ & & \\
\hline \multicolumn{4}{|c|}{ Tumor diameter $(\mathrm{cm})$} & 2.413 & 0.019 \\
\hline$\leq 7$ & 295 & | 47 (49.8) & $149(50.2)$ & & \\
\hline$>7$ & 44 & $30(68.2)$ & $14(3 \mid .8)$ & & \\
\hline \multicolumn{4}{|c|}{ Distant metastasis } & 6.280 & $<0.001$ \\
\hline Absent & 323 & $164(50.8)$ & $159(49.2)$ & & \\
\hline Present & 16 & $15(93.8)$ & I (6.2) & & \\
\hline \multicolumn{4}{|c|}{ Lymph node metastasis } & -3.758 & $<0.001$ \\
\hline Yes & 115 & $70(60.9)$ & $45(39.1)$ & & \\
\hline No & 224 & 89 (39.7) & $135(60.3)$ & & \\
\hline
\end{tabular}

Abbreviations: DcR3, decoy receptor 3; TNM, tumor-node-metastasis; NSCLC, non-small-cell lung cancer. 
Table 4 The status of DcR3 with respect to other clinicopathological parameters in SCLC

\begin{tabular}{|c|c|c|c|c|c|}
\hline$\overline{\text { SCLC }}$ & Case & DcR3-positive, n (\%) & e DcR3-negative, n (\%) & $Z$ & $\overline{P \text {-value }}$ \\
\hline \multicolumn{4}{|l|}{ Age (years) } & 0.181 & 0.858 \\
\hline$<60$ & 15 & $9(60.0)$ & $6(40.0)$ & & \\
\hline$\geqq 60$ & 11 & $7(63.6)$ & $4(36.4)$ & & \\
\hline \multicolumn{4}{|l|}{ Sex } & 1.000 & 0.352 \\
\hline Male & 21 & $12(57.1)$ & $9(42.9)$ & & \\
\hline Female & 5 & $4(80.0)$ & I (20.0) & & \\
\hline \multicolumn{4}{|l|}{ TNM } & 3.742 & 0.003 \\
\hline I or II & 13 & $6(46.2)$ & $7(53.8)$ & & \\
\hline III or IV & 10 & $10(100)$ & 0 & & \\
\hline \multicolumn{4}{|c|}{ Tumor diameter $(\mathrm{cm})$} & 3.240 & 0.005 \\
\hline$\leq 7$ & 19 & $12(63.2)$ & $7(36.8)$ & & \\
\hline$>7$ & 4 & $4(100)$ & 0 & & \\
\hline \multicolumn{6}{|c|}{ Distant metastasis } \\
\hline Absent & 23 & $16(69.6)$ & $7(30.4)$ & - & - \\
\hline Present & 0 & 0 & 0 & & \\
\hline \multicolumn{4}{|c|}{ Lymph node metastasis } & -2.898 & 0.012 \\
\hline Yes & 13 & $12(92.3)$ & I (7.7) & & \\
\hline No & 10 & $4(40.0)$ & $6(60.0)$ & & \\
\hline
\end{tabular}

Abbreviations: DcR3, decoy receptor 3; TNM, tumor-node-metastasis; SCLC, small-cell lung cancer.

(http://cbioportal.org) is an important part of TCGA. The data in cBioPortal include 20 cancer studies with more than 5,000 tumor samples. In this paper, the data regarding DcR3 expression in lung cancer were extracted and analyzed from cBioPortal. In addition, the original data regarding cancerous and noncancerous lung tissues were downloaded and analyzed.

\section{Statistical analysis}

SPSS20.0 was utilized for the statistical analysis. DcR3 expression among different tumor histological subtypes, pathological grading, and classifications were evaluated by Kruskal-Wallis $H$-test. Mann-Whitney $U$-test was used to compare DcR3 expression in different clinical features (age, sex, TNM stage, tumor size, distant metastasis, and lymph node metastasis). The relationships between DcR3 expression levels and clinicopathological parameters were assessed by Spearman's correlation. The receiver operator characteristic (ROC) curve was applied to determine the potential of DcR3 protein in lung cancer diagnosis. A $P$-value $<0.05$ was considered statistically significant (two sides).

\section{Results}

\section{Differential expression of DcR3 in lung cancer and normal lung tissues}

The positive signaling of DcR3, located in the cytoplasm of lung cancer cells or pulmonary epithelium of normal cells, is indicated by the formation of diffuse brown-yellow or dark brown color on immunohistochemical staining (Figure 1).
In this study, among the 365 cases of lung cancer, 196 cases were DcR3-positive (53.7\%), while positive DcR3 expression was observed in $15.4 \%$ of normal lung tissues (seven in 26 cases), which was significantly lower than that in lung cancer tissues (Table 1).

Then, DcR3 expression was assessed separately in SCLC and NSCLC. Higher levels of DcR3 expression were also found in both SCLC $(P=0.001)$ and NSCLC $(P=0.008)$, as compared to that in noncancerous lung tissues. However, only a slightly higher expression of DcR3 was observed in $\operatorname{SCLC}(73.1 \%)$ compared to that in $\operatorname{NSCLC~}(53.1 \%, P=0.039)$ tissues. We also investigated the expression of DcR3 in different histologic types of NSCLC. We found that the expression of DcR3 was higher in adenocarcinoma $(56.7 \%$, $P=0.005)$, squamous cell carcinoma $(48.6 \%, P=0.031)$, adenosquamous carcinoma $(60.7 \%, P=0.012)$, and undifferentiated carcinoma $(75 \%, P=0.013)$ than in normal lung tissues (Table 1). In addition, ROC curve was applied to analyze the diagnostic value of DcR3 level in lung cancer. The area under curve (AUC) of DcR3 was 0.637 (95\% confidence interval $[\mathrm{CI}] 0.531-0.742, P=0.02)$.

\section{Relationships between DcR3 protein and other clinicopathological parameters in lung cancer}

Relationships between DcR3 protein and other clinicopathological parameters in lung cancer tissues were further investigated. In all 66 advanced-stage (III/IV) lung cancer cases, the positive expression of DcR3 (77.3\%) was markedly higher 


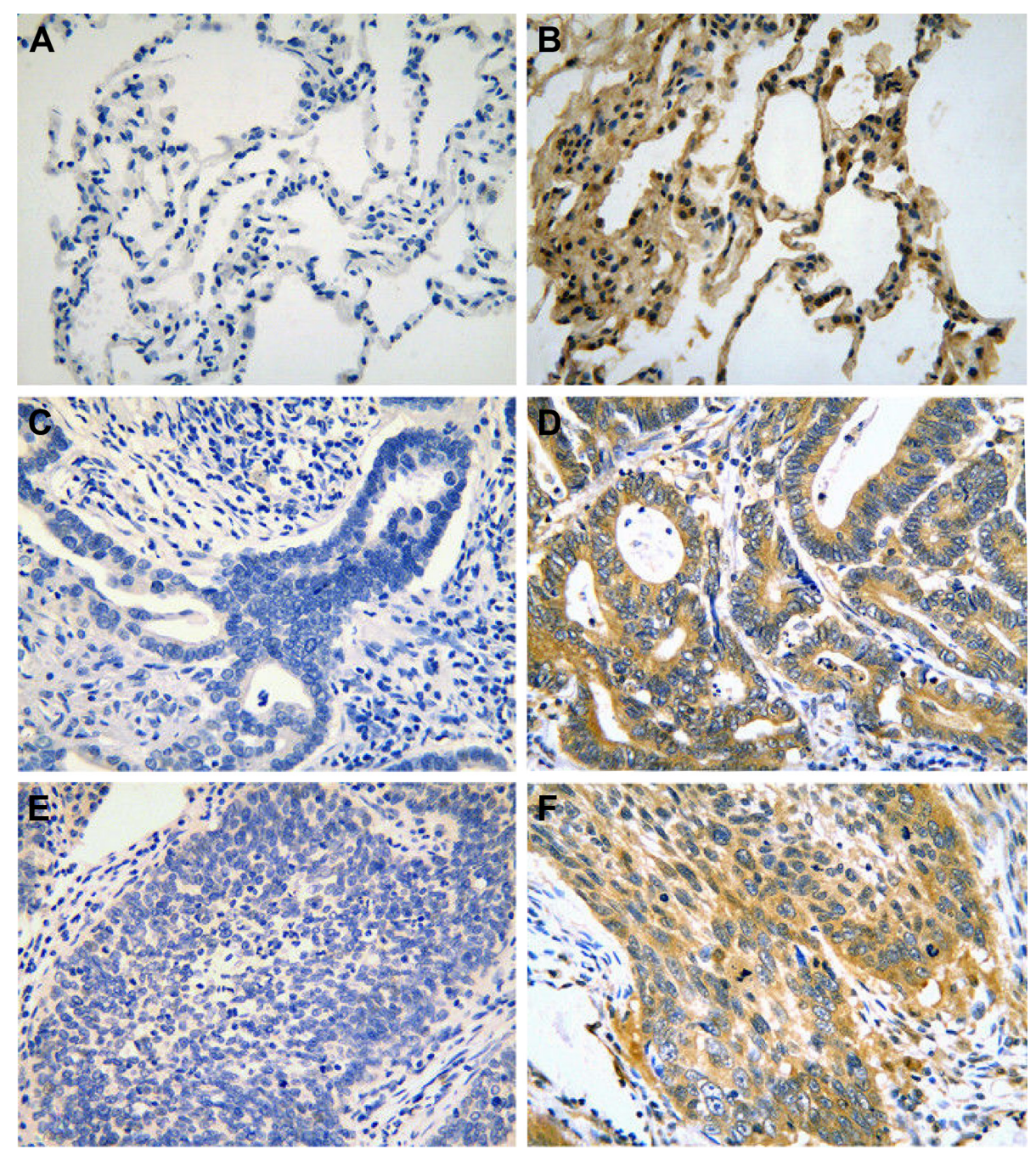

Figure I Immunohistochemical staining of DcR3 expression in lung cancers.

Notes: DcR3 signaling was predominantly observed in the cytoplasm of lung cancer cells. Negative staining in normal lung tissue (A); positive expression in normal lung tissue (B); negative staining in lung adenocarcinoma (C); positive expression in lung adenocarcinoma (D); negative staining in lung squamous carcinoma (E); positive expression in lung squamous carcinoma (F). Magnification, 400x.

Abbreviation: DcR3, decoy receptor 3.

compared to that in early stages (I and II, $48.2 \%, P<0.001$ ). As for tumor diameter, the positive expression of DcR3 was found to be higher in tumors of larger size $(>7 \mathrm{~cm}, 70.6 \%)$ than in those of smaller size $(\leq 7 \mathrm{~cm}, 51.0 \%, P=0.007)$. Concerning distal metastasis, positive expression of DcR3 was found to be significantly higher in distant metastasis tumor group $(15 / 16,93.8 \%)$ than in group showing no metastasis $(180 / 349,51.6 \%, P<0.001)$. Additionally, the positive expression of DcR3 was observed to be significantly higher in patients with lymph node metastasis tumors $(102 / 128$, $79.7 \%$ ) than in those with no lymph node metastasis $(96 / 237$, $40.5 \%, P<0.001$, Table 2). Spearman's test was performed to assess the correlations between DcR3 and lung cancer, which showed that consistent correlations existed between DcR3 and TNM stage $(r=0.225, P<0.001)$, tumor diameter
( $r=0.137, P=0.009)$, distant metastasis $(r=0.173, P=0.001)$, and lymph node metastasis $(r=0.375, P<0.001)$. We also calculated the correlation between the differential expression of DcR3 and age and sex, but no significant difference was observed (Tables 2 and 5).

Further, we studied the clinical contribution of DcR3 in the subtypes of SCLC and NSCLC. In NSCLC, a consistent correlation between TNM stage, tumor diameter, distal and lymph node metastasis, and DcR3 expression was found. Specifically, positive expression of DcR3 was found in 41 out of 53 NSCLC cases in advanced stages (III and IV, 77.4\%), clearly higher than in early stages (I and II, 138/286, 48.3\%, $P<0.001)$. The positive expression of DcR3 was upregulated in larger tumors $(>7 \mathrm{~cm}, 30 / 44,68.2 \%)$ than in smaller ones ( $\leq 7 \mathrm{~cm}, 147 / 295,48.3 \%, P=0.019$ ). The positive expression 
Table $\mathbf{5}$ The correlation between DcR3 expression and other clinicopathological parameters in lung cancer, SCLC, and NSCLC

\begin{tabular}{llll}
\hline DcR3 expression & Lung cancer & SCLC & NSCLC \\
\hline Age & & & \\
$\quad R$-value & -0.019 & 0.037 & 0.036 \\
$\quad P$-value & 0.713 & 0.858 & 0.511 \\
$\begin{array}{l}\text { Sex } \\
R \text {-value }\end{array}$ & 0.066 & 0.185 & 0.091 \\
$\quad P$-value & 0.210 & 0.365 & 0.095 \\
TNM & & & \\
$R$-value & 0.225 & 0.580 & 0.212 \\
$P$-value & $<0.001$ & 0.004 & $<0.001$ \\
Tumor diameter $(\mathrm{cm})$ & & & \\
$\quad R$-value & 0.137 & 0.303 & 0.124 \\
$\quad P$-value & 0.009 & 0.159 & 0.022 \\
Distant metastasis & & & \\
$\quad R$-value & 0.173 & $\mathrm{NA}$ & 0.183 \\
$\quad P$-value & 0.001 & $\mathrm{NA}$ & 0.001 \\
Lymph node metastasis & & & \\
$\quad R$-value & 0.375 & 0.564 & 0.201 \\
$P$-value & $<0.001$ & 0.005 & $<0.001$ \\
\hline
\end{tabular}

Abbreviations: DcR3, decoy receptor 3; TNM, tumor-node-metastasis; SCLC, smallcell lung cancer; NSCLC, non-small-cell lung cancer; NA, not applicable.

of DcR3 was remarkably higher in distant metastasis tumor group $(15 / 16,93.8 \%)$ than in the group with no distant metastasis $(164 / 323,50.8 \%, P<0.001)$. Furthermore, when lymph node metastasis was concerned, the positive expression of DcR3 was found in 70 among 115 NSCLC cases with lymph node metastasis $(60.9 \%)$, which was significantly higher than in those without $(39.7 \%, 89 / 224, P<0.001$, Table 3). Spearman's test showed that there were consistent correlations between DcR3 expression and TNM stage ( $r=0.212, P<0.001)$, tumor diameter $(r=0.124, P=0.022)$, distant metastasis $(r=0.183, P=0.001)$, and lymph node metastasis $(r=0.201, P<0.001$, Table 5). No significant difference was found in groups with respect to age and sex (Tables 3 and 5).

Simultaneously, in SCLC, DcR3 expression was also found to be associated with TNM stage $(r=0.580, P=0.004)$ and lymph node metastasis $(r=0.564, P=0.005)$. The positive expression of DcR3 was found to be higher in advanced stages (III and IV, 100\%) than in early stages (I and II, $6 / 13,46.2 \%, P=0.003)$. With regard to tumor diameter, the positive expression of DcR3 was remarkably higher in larger tumors $(>7 \mathrm{~cm}, 12 / 19,63.2 \%)$ than in smaller ones $(\leq 7 \mathrm{~cm}$, $4 / 4,100 \%, P=0.005)$. In addition, the positive expression of DcR3 was found in 12 out of 13 SCLC cases with lymph node metastasis $(92.3 \%)$, clearly higher than in those with no lymph node metastasis $(4 / 10,40.0 \%, P=0.012$, Table 4$)$. No significant difference was found with regard to age, sex, and distant metastasis (Tables 4 and 5).

\section{Relationships between DcR3 expression and clinical parameters in lung cancer according to the data from TCGA}

To further elucidate the clinical role of DcR3 in the survival of lung cancer patients, we searched the data of 521 adenocarcinoma and 504 squamous cell carcinoma cases in TCGA. Analysis of TCGA data by cBioPortal (http:// www.cbioportal.org/public-portal/) demonstrated that overall, DcR3 was highly expressed in lung cancer, and the upregulated expression of DcR3 was observed in 18\% of all adenocarcinoma cases (Figure 2). We further confirmed this correlation at the mRNA level. We found that DcR3 mRNA was overexpressed in adenocarcinoma patients (Figure 3). Similarly to adenocarcinoma, upregulated expression of DcR3 was also found in $21 \%$ of all squamous cell carcinoma samples (Figure 4). Further, we confirmed this correlation at the mRNA level. A high expression of DcR3 mRNA was found in squamous cell carcinoma cases (Figure 5). In order to further explore the clinical value of DcR3 in lung cancer, including adenocarcinoma and squamous cell carcinoma, the original data were downloaded and analyzed from TCGA datasets, and we found that DcR3 was overexpressed in both adenocarcinoma and squamous cell carcinoma tissues than in noncancerous lung tissues (all $P<0.0001$, Figure 6). We also compared the expression between adenocarcinoma

Case set: tumors with mRNA data (RNA Seq V2): all samples with mRNA expression data (515 patients/517 samples)
Show all samples

Altered in $93(18 \%)$ of 517 cases/patients

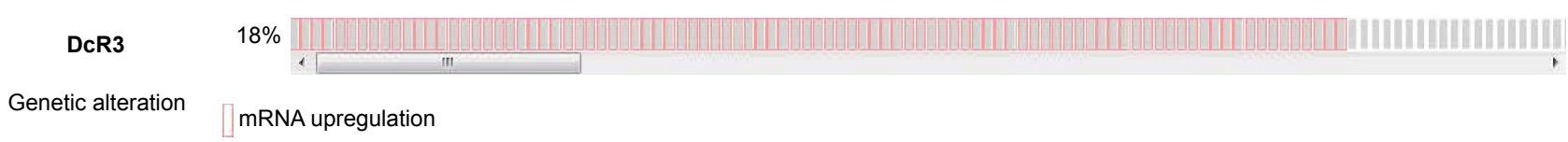

Figure 2 The upregulated expression of DcR3 in all samples of adenocarcinoma. Abbreviations: DcR3, decoy receptor 3; mRNA, messenger RNA. 


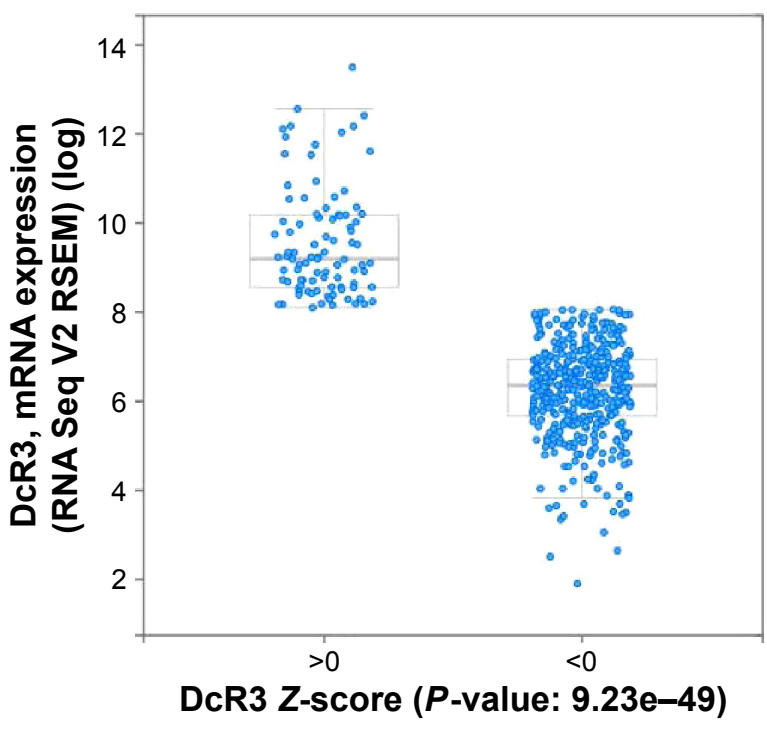

Figure 3 The expression of DcR3 mRNA in all samples of adenocarcinoma. Abbreviations: DcR3, decoy receptor 3; mRNA, messenger RNA; RSEM, RNAseq by expectation maximization.

and squamous cell carcinoma tissues, but no significant difference was noted between these two groups $(294.5 \pm 36.23$ vs $288.3 \pm 29.12, P=0.894)$.

In addition, the ROC curve revealed that the AUC of DcR3 was 0.726 (95\% CI 0.644-0.788) for lung adenocarcinoma patients and 0.647 (95\% CI $0.566-0.728)$ for squamous cell carcinoma patients, which was applied to analyze the diagnostic value of DcR3 level in lung cancer. Interestingly, we also investigated the relationship between DcR3 level and patients' survival. The upregulated expression of DcR3 was associated with overall survival $(P<0.001$, $98.8 \pm 9.34$ vs $39.7 \pm 4.22)$ and disease-free survival of adenocarcinoma patients $(P<0.001,133.0 \pm 12.92$ vs $47.0 \pm 8.34$, Figures 7 and 8), which indicated that DcR3 could influence the prognosis.

\section{Discussion}

Lung cancer is a general and frequently occurring disease that threatens human health seriously. With the acceleration of urbanization, the incidence and mortality of lung cancer has also been increasing. So far, a variety of studies related to genes involved in lung cancer have been carried out. ${ }^{25,26}$ For instance, Deben et $\mathrm{al}^{25}$ explored the role of p53 in carcinogenesis and they found that the expression of $\mathrm{p} 53$ could be used to evaluate the prognostic and predictive significance in lung cancer patients. Garajova et $\mathrm{a}^{26}$ found that c-Met could be a target for personalized therapy, but no report was available to explain the correlation between the expression of DcR3 and lung cancer. In this study, we attempted to evaluate the relationship between DcR3 and lung cancer and further investigate the prospective role of DcR3 expression in the prediction and diagnosis of lung cancer.

In our present study, we explored the DcR3 expression in lung cancer and normal lung samples by tissue microarray and IHC. We found that DcR3 expression in lung cancer, including SCLC and NSCLC, was clearly higher compared to that in the normal lung tissues. The ROC curve indicated that DcR3 protein might have a moderate diagnostic value for lung cancer (AUC $=0.637$ ). Also, in the study of the correlation between the expression of DcR3 and some clinicopathological parameters, we found that DcR3 expression was remarkably related to the deterioration of the disease, including both of NSCLC and SCLC. The higher expression of DcR3 might prompt an advanced stage or larger tumor size or metastasis. Also, the low survival rate showed that the overexpression of DcR3 might indicate a poor prognosis. We thus could draw a conclusion that DcR3 might act as a molecular biomarker in lung cancer, and our study confirms that DcR3 might be involved in the tumorigenesis and deterioration of lung cancer, although further verification is required. In other reports, Huang et al studied 125 glioma patients and 18 normal brain cases and found that the higher expression of DcR3 might be related to tumor cell differentiation and proliferation. ${ }^{27}$ Zhou et $\mathrm{al}^{28,29}$ investigated the relationship between high expression of DcR3 and prognosis of pancreatic carcinoma. They found that high expression of DcR3 could predict the prognosis of pancreatic carcinoma and that the DcR3 level in sera could be used for the early

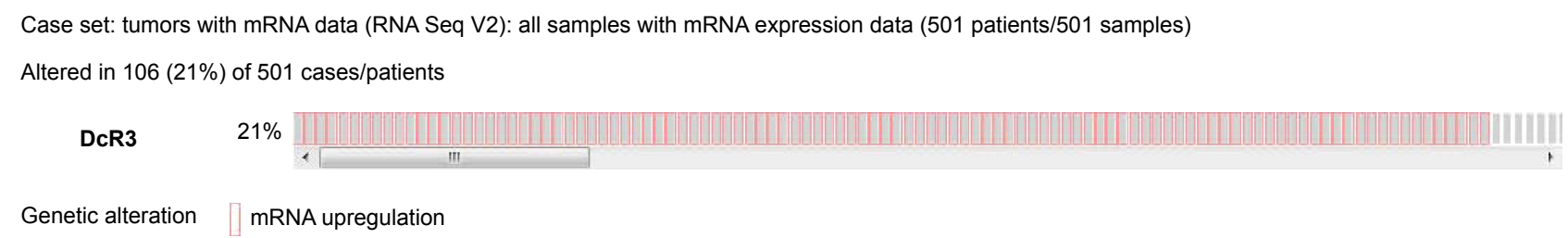

Figure 4 The upregulated expression of DcR3 in all samples of squamous cell carcinoma.

Abbreviations: DcR3, decoy receptor 3; mRNA, messenger RNA. 


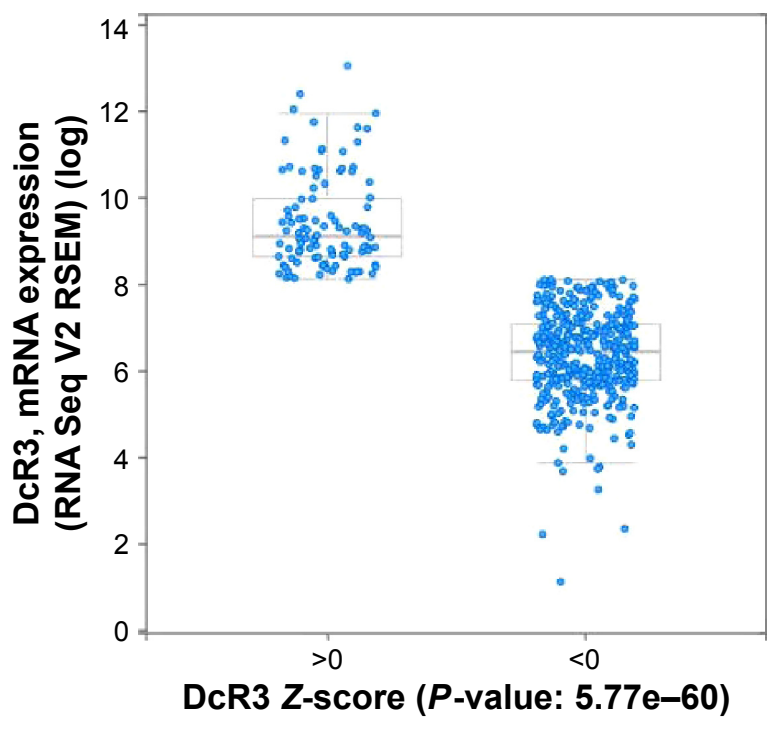

Figure $\mathbf{5}$ The expression of DcR3 mRNA in all samples of squamous cell carcinoma. Abbreviations: DcR3, decoy receptor 3; mRNA, messenger RNA; RSEM, RNAseq by expectation maximization.

diagnosis and prognostic judgment of pancreatic carcinoma. In addition, TCGA database was used to verify the DcR3 expression in lung cancer. Consistent with our results, DcR3 was found to be highly expressed in lung cancer patients according to the data from TCGA. All the aforementioned results demonstrated that DcR3 can be used for the early diagnosis and prognostic judgment of lung cancer.

The role of DcR3 in lung cancer has been preliminarily studied by several groups. In agreement with our results, Pitti et $\mathrm{al}^{14}$ reported that the $D c R 3$ gene was highly expressed in lung cancers. They studied 35 primary lung cancer cases and found that DcR3 played its role by binding specifically to FasL, thereby inhibiting its activity. DcR3 has also been reported to correlate with FasL in some malignant tumors, such as pancreatic cancer and hepatocellular carcinoma..$^{30,31}$ FasL is an important factor involved in the regulation of the immune response. Also, the FasL-Fas receptor could act as a key physiological regulator in programmed cell death. ${ }^{32}$ Zhang et $\mathrm{al}^{30}$ found that DcR3 could suppress FasL-induced apoptosis via ERK1/2 pathway in pancreatic cancer cells, and reported that DcR3 could enhance ERK1/2 phosphorylation and oppose FasL signaling. In our previous study of DcR3 in glioma, we obtained results consistent with those reported by Zhang et al that DcR3 could cause an effect on cell growth suppression and apoptosis induction in glioma cells. ${ }^{19}$ In our present study, we envision that the overexpression of DcR3 could also be correlated to Fasl via ERK to inhibit growth and induce apoptosis of lung cancer cells. But the authentic pathway or the mechanism of action of DcR3 on the biological function of lung cancer needs further exploration.

In addition, our previous study found that DcR3 functions as a bridge between miR-152 and hepatocellular carcinoma. ${ }^{33}$ Besides miR-152, DcR3 could be the target gene for other miRNAs through bioinformatics softwares. miR-148 and miR-340 were predicted by five different algorithms, including TargetScan, miRDB, DIANA-microT, miRanda, and miRWalk (data not shown). However, the molecular mechanism underlying the role of DcR3 in lung cancer is still unclear, and further in vitro and in vivo investigations will be required in the future.

\section{Conclusion}

DcR3 expression, as assessed by IHC, is upregulated in lung cancer, including SCLC and NSCLC. The expression of DcR3 protein is positively correlated with the deterioration
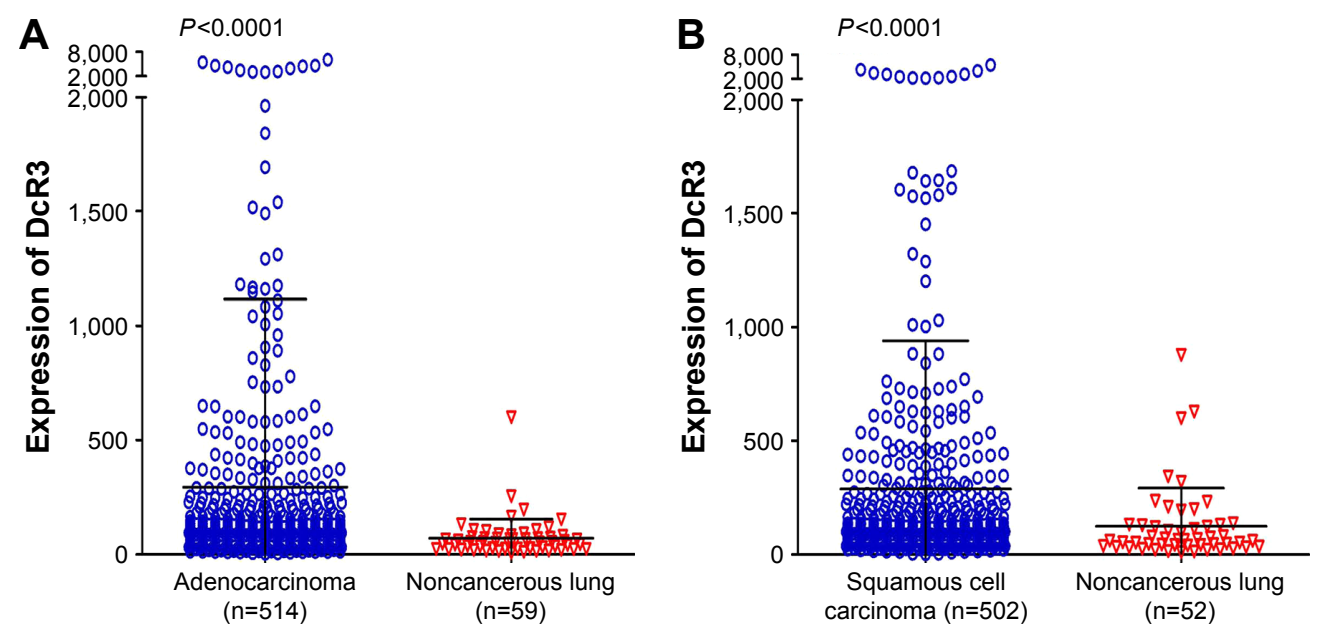

Figure 6 Clinical significance of DcR3 in lung adenocarcinoma and squamous cell carcinoma based on The Cancer Genome Atlas database. Notes: The expression of DcR3 in adenocarcinoma (A) and squamous cell carcinoma (B).

Abbreviation: DcR3, decoy receptor 3. 


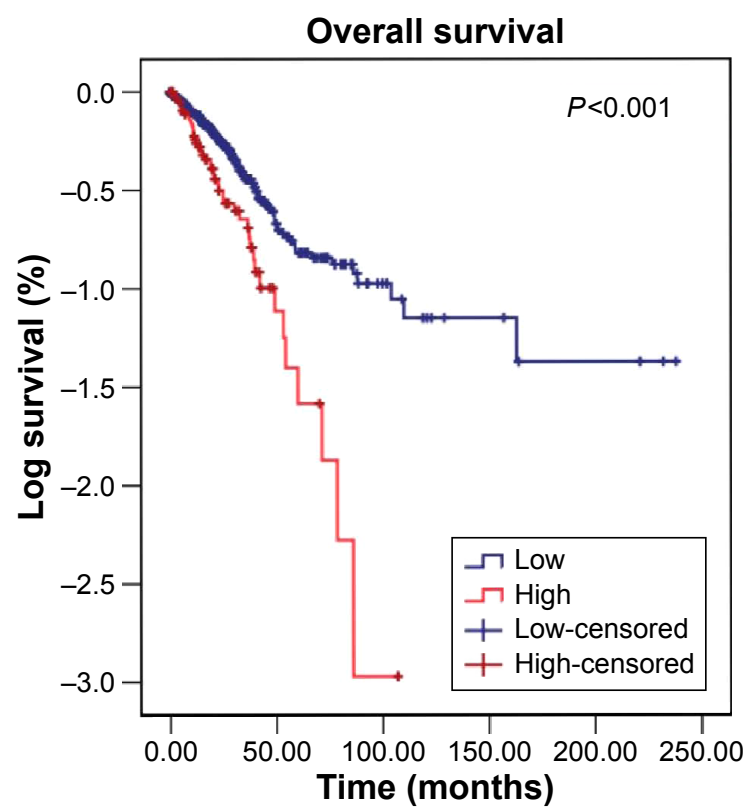

Figure 7 The expression of DcR3 is associated with overall survival of adenocarcinoma patients.

Notes: Overall survival Kaplan-Maier estimates for gene signature (DcR3, log-rank test, $P<0.001$ ). Red indicates cases with high expression of DcR3.

Abbreviation: DcR3, decoy receptor 3.

and poor prognosis of lung cancer patients. Together with previous reports, the current study strongly suggests that DcR3 might play a crucial role in the evolution and progression of lung cancer. Further experiments are expected to explore the prospective molecular mechanism of DcR3 in lung cancer.

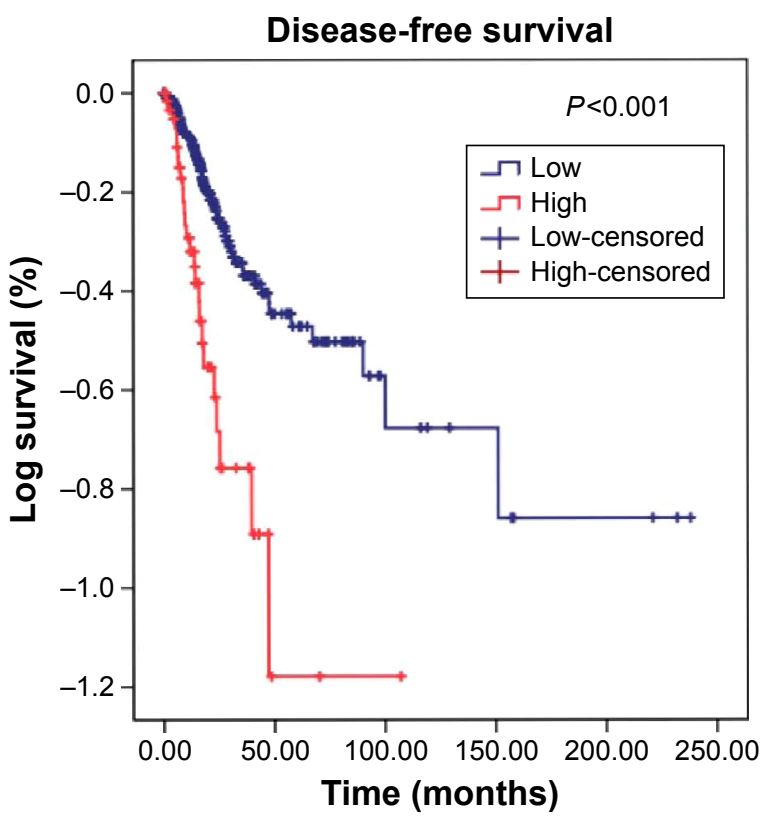

Figure 8 The expression of DcR3 is associated with disease-free survival of adenocarcinoma patients.

Notes: Overall survival Kaplan-Maier estimates for gene signature (DcR3, log-rank test $P<0.00$ I). Red indicates cases with high expression of DcR3.

Abbreviation: DcR3, decoy receptor 3.

\section{Acknowledgments}

The study was supported by the fund of National Natural Science Foundation of China (NSFC81360327, NSFC81560469), Guangxi Natural Science Foundation (2015GXNSFCA139009), Scientific Research Project of the Guangxi Education Agency (KY2015LX062), Guangxi Graduate Innovation Project (YCSZ2015106), and Guangxi Provincial Health Bureau Scientific Research Project (Z2013201, Z2014055). The funders had no role in study design, data collection and analysis, decision to publish, or preparation of the manuscript.

\section{Disclosure}

The authors report no conflicts of interest in this work.

\section{References}

1. Xu YJ, Du Y, Fan Y. Long noncoding RNAs in lung cancer: what we know in 2015. Clin Transl Oncology. 2015. Epub 2015 Dec 15.

2. Kang CG, Lee HJ, Kim SH, Lee EO. Zerumbone Suppresses osteopontin-induced cell invasion through inhibiting the FAK/AKT/ ROCK pathway in human non-small cell lung cancer A549 cells. $J$ Nat Product. 2016;79(1):156-160.

3. Torre LA, Bray F, Siegel RL, Ferlay J, Lortet-Tieulent J, Jemal A. Global cancer statistics, 2012. CA: Cancer J Clin. 2015;65(2):87-108.

4. Chen G, Umelo IA, Lv S, et al. miR-146a inhibits cell growth, cell migration and induces apoptosis in non-small cell lung cancer cells. PloS One. 2013;8(3):e60317.

5. Yilmaz A, Damadoglu E, Salturk C, Okur E, Tuncer LY, Halezeroglu S. Delays in the diagnosis and treatment of primary lung cancer: are longer delays associated with advanced pathological stage? Upsala J Med Sci. 2008;113(3):287-296.

6. Pfister DG, Johnson DH, Azzoli CG, et al. American Society of Clinical Oncology treatment of unresectable non-small-cell lung cancer guideline: update 2003. J Clin Oncol. 2004;22(2):330-353.

7. Morishige T, Yoshioka Y, Inakura $\mathrm{H}$, et al. Creation of a lysine-deficient LIGHT mutant with the capacity for site-specific PEGylation and low affinity for a decoy receptor. Biochem Biophys Res Commun. 2010;393(4): 888-893.

8. Connor JP, Felder M, Kapur A, Onujiogu N. DcR3 binds to ovarian cancer via heparan sulfate proteoglycans and modulates tumor cells response to platinum with corresponding alteration in the expression of BRCA1. BMC Cancer. 2012;12:176.

9. Wang W, Zhang M, Sun W, et al. Reduction of decoy receptor 3 enhances TRAIL-mediated apoptosis in pancreatic cancer. PloS One. 2013;8(10): e74272.

10. Yang D, Fan X, Yin P, et al. Significance of decoy receptor 3 (Dcr3) and external-signal regulated kinase $1 / 2($ Erk1/2) in gastric cancer. $B M C$ Immunol. 2012;13:28.

11. Weissinger D, Tagscherer KE, Macher-Goppinger S, Haferkamp A, Wagener N, Roth W. The soluble decoy receptor 3 is regulated by a PI3K-dependent mechanism and promotes migration and invasion in renal cell carcinoma. Mol Cancer. 2013;12.

12. Chen G, Rong M, Luo D. TNFRSF6B neutralization antibody inhibits proliferation and induces apoptosis in hepatocellular carcinoma cell. Pathol Res Pract. 2010;206(9):631-641.

13. Arakawa Y, Tachibana O, Hasegawa M, Miyamori T, Yamashita J, Hayashi Y. Frequent gene amplification and overexpression of decoy receptor 3 in glioblastoma. Acta Neuropathol. 2005;109(3):294-298.

14. Pitti RM, Marsters SA, Lawrence DA, et al. Genomic amplification of a decoy receptor for Fas ligand in lung and colon cancer. Nature. 1998; 396(6712):699-703

15. Roth W, Isenmann S, Nakamura M, et al. Soluble decoy receptor 3 is expressed by malignant gliomas and suppresses CD95 ligand-induced apoptosis and chemotaxis. Cancer Res. 2001;61(6):2759-2765. 
16. Sung HY, Wu HG, Ahn JH, Park WY. Dcr3 inhibit p53-dependent apoptosis in gamma-irradiated lung cancer cells. Int J Rad Biol. 2010; 86(9):780-790.

17. Wu Y, Han B, Sheng H, et al. Clinical significance of detecting elevated serum DcR3/TR6/M68 in malignant tumor patients. Int J Cancer. 2003; 105(5):724-732.

18. Huang S, Chen G. Overexpression of DcR3 and its significance on tumor cell differentiation and proliferation in glioma. Scientific World Journal. 2014;2014:605236.

19. Ruan Y, Huang S, He D, Gopaul R, Li Z, Chen G. Effect of TNFRSF6B neutralization antibody on cell growth suppression and apoptosis induction in glioma cells. Neoplasma. 2015;62(4):574-581.

20. Chen G, Luo D. Over-expression of decoy receptor 3 in gastric precancerous lesions and carcinoma. Upsala J Med Sci. 2008;113(3):297-304.

21. Yang M, Chen G, Dang Y, Luo D. Significance of decoy receptor 3 in sera of hepatocellular carcinoma patients. Upsala J Med Sci. 2010;115(4): 232-237.

22. Bornstein S, Schmidt M, Choonoo G, et al. IL-10 and integrin signaling pathways are associated with head and neck cancer progression. $B M C$ Genomics. 2016;17(1):38.

23. Cerami E, Gao J, Dogrusoz U, et al. The cBio cancer genomics portal: an open platform for exploring multidimensional cancer genomics data. Cancer Discov. 2012;2(5):401-404.

24. Gao J, Aksoy BA, Dogrusoz U, et al. Integrative analysis of complex cancer genomics and clinical profiles using the cBioPortal. Sci Signal. 2013;6(269):pl1.

25. Deben C, Deschoolmeester V, Lardon F, Rolfo C, Pauwels P. TP53 and MDM2 genetic alterations in non-small cell lung cancer: evaluating their prognostic and predictive value. Crit Rev Oncol/Hematol. 2016;99:63-73.
26. Garajova I, Giovannetti E, Biasco G, Peters GJ. c-Met as a target for personalized therapy. Transl Oncogenomics. 2015;7(Suppl 1):13-31.

27. Huang S, Chen G, Dang Y, Chen LH. Overexpression of DcR3 and its significance on tumor cell differentiation and proliferation in glioma. Scientific WorldJournal. 2014;2014:605236.

28. Zhou J, Song S, Li D, et al. Decoy receptor 3 (DcR3) overexpression predicts the prognosis and $\mathrm{pN} 2$ in pancreatic head carcinoma. World J Surg Oncol. 2014;12:52.

29. Zhou J, Song SD, Li DC, Zhou J, Zhu DM, Zheng SY. Clinical significance of expression and amplification of the DcR3 gene in pancreatic carcinomas. Asian Pac J Cancer Prev. 2012;13(2):719-724.

30. Zhang Y, Li D, Zhao X, et al. Decoy receptor 3 suppresses FasL-induced apoptosis via ERK1/2 activation in pancreatic cancer cells. Biochem Biophys Res Commun. 2015;463(4):1144-1151.

31. Li W, Zhang C, Chen C, Zhuang G. Correlation between expression of DcR3 on tumor cells and sensitivity to FasL. Cell Mol Immunol. 2007;4(6): 455-460.

32. Kim SK, Yoon YD, Park YS, Seo JT, Kim JH. Involvement of the Fas-Fas ligand system and active caspase-3 in abnormal apoptosis in human testes with maturation arrest and Sertoli cell-only syndrome. Fertil Steril. 2007;87(3):547-553.

33. Dang YW, Zeng J, He RQ, Rong MH, Luo DZ, Chen G. Effects of miR-152 on cell growth inhibition, motility suppression and apoptosis induction in hepatocellular carcinoma cells. Asian Pac J Cancer Prev. 2014; 15(12):4969-4976.
OncoTargets and Therapy

\section{Publish your work in this journal}

OncoTargets and Therapy is an international, peer-reviewed, open access journal focusing on the pathological basis of all cancers, potential targets for therapy and treatment protocols employed to improve the management of cancer patients. The journal also focuses on the impact of management programs and new therapeutic agents and protocols on

\section{Dovepress}

patient perspectives such as quality of life, adherence and satisfaction The manuscript management system is completely online and includes a very quick and fair peer-review system, which is all easy to use. Visit http://www.dovepress.com/testimonials.php to read real quotes from published authors. 